As part of the 50th birthday celebration, we are arranging to reprint 12 papers, the Editor's Choice, which have appeared in previous issues of the fournal. They have been chosen partly to illustrate the range and scope of the fournal over the years and partly because they are or were important in their day. More significantly, they have been chosen because they exemplify some of the best in scientific writing and can all be read with great pleasure and all who wish to communicate their observations, their ideas, or their enthusiasms would do well to study them and learn from them.

We will publish one paper each month through the year and they will appear in the order in which they were originally published.

\title{
Mesothelioma of pleura and peritoneum following exposure to asbestos in the London area
}

\author{
by Muriel L Newhouse and Hilda Thompson
}

\section{(British fournal of Industrial Medicine 1965;22:262-9)}

Molly Newhouse holds a special place in the affections of practitioners of occupational medicine and many now highly placed were students of hers at the TUC Centenary Institute of Occupational Health at the London School of Hygiene and Tropical Medicine. Her principal research interests were in skin disease and in diseases related to exposure to asbestos. The present paper describes a study investigating the aetiology of mesothelioma in a group of 83 patients, 42 men and 41 women. Among the men occupational exposure to asbestos was common, involving more than half the group. Of the women, there were 10 with occupational exposure but seven with domestic exposure. "The most usual history was of the wife who washed her husband's ... work clothes. In one instance ... the husband came home 'white with asbestos' every day for three or four years and his wife brushed him down." There were also two men who, when boys of 8 or 9 , had lived in the house with a sister who had worked in an asbestos factory.

Of even more interest in this study were cases of neighbourhood exposure. There were 11 patients who have lived within half a mile of an asbestos factory, a number significantly greater than among a control group of patients without mesothelioma. This suggested that neighbourhood exposure was the important source in these cases. The authors seemed to be reluctant to stress these findings, however, contenting themselves merely to say that more evidence was required. There are a good many others who, faced with the same findings, would have been considerably less reticent. 